\title{
Study and Application of Guide-line Method for Power Generation Operation of Inter-basin Reservoirs
}

\author{
Weijiang Zhu ${ }^{1, a}$, Binqi $\mathrm{Hu}^{1, \mathrm{~b}}$, Yonggang $\mathrm{Wu}^{2, \mathrm{c}}$, Jia $\mathrm{Chen}^{2, \mathrm{~d}}$, Tao Cheng ${ }^{1, \mathrm{e}}$ and \\ Jianping Liu ${ }^{1, f}$ \\ ${ }^{1}$ Hunan Electric Power Company Dispatching and Communication Bureau, Changsha 410007, \\ China. \\ ${ }^{2}$ School of Hydropower and Information Engineering, Huazhong University of Science and \\ Technology, Wuhan 430074, China. \\ azhuwj2@hn.sgcc.com.cn, bhubq@hn.sgcc.com.cn, 'cygangwu@163.com, ${ }^{d}$ cj0370@126.com, \\ echengt@hn.sgcc.com.cn, 'liujping@hn.sgcc.com.cn
}

Keywords: inter-basin, multi-reservoir, coordination strategy, high water-level, minimum spillage, discriminant criterion method.

Abstract. In order to overcome the defects of emptying reservoir and abandoning water of discriminant criterion method in inter-basin reservoirs operation, this paper analyses the relationships between $\mathrm{K}$ value and its calculating time period, takes the minimum spillage storage line as the guide line of reservoir running that is used to determine the terminal time for calculating reservoir's $\mathrm{K}$ value and accurately assesses energy loss of inflow and spill energy change caused by reservoir storing and supplying. On this basis, a coordination strategy for generation decision between inter-basin reservoirs is presented. A inter-basin reservoir system consist of four hydropower plants in Hunan power grid is taken for example and the simulating results show that the presented method has high solution efficiency as well as good search ability, made up for the shortcomings of the discriminant criterion method and shows advantages on both optimization degree of scheduling results and execution time of algorithm over dynamic programming successive approximation (DPSA). The research results provide a new ideas for solving the power generation dispatch problem of inter-basin reservoirs.

\section{Introduction}

Hydrological characteristic of reservoir varies in different river basins. It can improve the entire benefits of multi-reservoir system to implement compensation scheduling among different inter basins by making full use of the diversity of hydrological characteristic of different reservoirs [1-2].

In study and application, dynamic programming (DP) [3-5] and the criminate criterion method [6-10] are commonly used to solve the power generation operation problem of multi-reservoir. DP applies to solve the multi-stage decision problem and is widely used in the area of power generation of reservoir, but the largest technical obstacle of DP is "dimension curse" in sense that the calculation and memory requirements of DP increase exponentially with the number of reservoirs. In order to overcome the "dimension curse" of DP, many scholars research different dimension-reducing technologies and propose different improved methods of DP such as discrete differential dynamic programming (DDDP) [11-12], dynamic programming with successive approximation (DPSA) [13-14] and progressive optimality algorithm (POA) [15-16]. Although these methods alleviate the "dimension curse" of DP to a certain extent, they achieve it at the expense of optimality or precision of solution. The criminate criterion method determines the order of storing water or supplying water of reservoirs by the principle of minimizing energy loss of inflow which is measured by K-value of reservoir. K-value is an energy index that represents energy loss of inflow per increasing unit power output. It follows the regular that reservoir with large $\mathrm{K}$-value has priority to storing water and reservoir with small $\mathrm{K}$-value always has priority to supply water. The criminate criterion method has clear physical meaning but it usually causes to empty the reservoir and produce spillage which is unfavorable to make full use of hydropower resources [17]. In order to overcome the defects of the 
criminate criterion method, literatures [17] and [18] combine the criminate criterion method with reservoir operation chart to control the operation process of multi-reservoir system. It acquires certain effect.

Analysis indicates that storing water or supplying water will change the energy loss of inflow and spill energy of reservoir in following several time periods; however, the criminate criterion method only takes account of energy loss of the time span from the current time to the end of storing time period or supplying time period. This is why the criminate criterion method may produce some irrational phenomena in practical application.

Given this, this paper introduces reservoir guide-line to accurately determine the end time for calculating K-value of reservoir based on evaluating energy loss of inflow and spill energy caused by storing water or supplying water of reservoir. In the later parts of this paper, the guide-line method is applied to solve the power generation operation problem of multi-reservoir system.

\section{Problem Description}

Power generation operation of multi-reservoir often takes maximizing power generation as the optimization objective. The constraints conclude water balance equation, minimum and maximum water level, minimum and maximum release, minimum and maximum power output, the minimum total power output of multi-reservoir system, reservoir boundary conditions and so on. Details are given by literatures [0].

\section{The Guide-line Method for Power Generation Operation of Multi-reservoirs of Inter Basins}

Knowledge and Experiences for Power Generation Operation of Reservoir [0]-[0]. The energy for power generation of reservoir derives from river runoff. Some of the mechanical energy of river runoff is converted to electricity by hydropower plant and the others is lost. The lost energy mainly includes energy loss of inflow and spill energy. The expressions of energy loss of inflow and spill energy of reservoir in time period $t$ are as follows.

$$
\left\{\begin{array}{l}
E_{\text {loss }, t}=\left(\bar{Z}_{\mathrm{u}}-Z_{\mathrm{u}, t}\right) \cdot I_{t} \cdot \Delta t \\
E_{\mathrm{spill}, t}=\left(Z_{\mathrm{u}, t}-\underline{Z}_{\mathrm{d}}\right) \cdot Y_{t} \cdot \Delta t
\end{array}\right.
$$

Where $E_{\text {loss }, t}$ and $E_{\text {spill, } t}$ denote energy loss of inflow and spill energy of reservoir in time period $t$, in $\mathrm{kW} \cdot \mathrm{h} ; Z_{\mathrm{u}, t}$ is average water level of reservoir in time period $t$, in $\mathrm{m} ; \bar{Z}_{\mathrm{u}}$ and $\underline{Z}_{\mathrm{d}}$ denote the normal water level and the minimum tail water level of reservoir, in $\mathrm{m} ; I_{t}$ and $Y_{t}$ are inflow and spillage of reservoir in time period $t$, in $\mathrm{m}^{3} / \mathrm{s} ; \Delta t$ denotes the length of a time period, in $\mathrm{s}$.

According to the law of the conservation of energy, reducing energy loss can improve power generation when the total energy (the energy carried by river runoff) is constant. From Eq. (1), it can be seen that reservoir should keep running at a high water level because the higher the water level is, the less the energy loss of inflow is. Similarly, from Eq. (1), it can be seen that reservoir should avoid abandoning water as far as possible because the less the spillage is, the less the spill energy is. From the above analysis, three principles for power generation operation of reservoir are concluded as follows.

1) Reservoir should generate at a high water level (call it "high water level" principle).

2) Reservoir should reduce spillage as far as possible (call it "reducing spillage" principle).

3) Reservoir should delay the spill time as far as possible when abandoning water cannot be avoided (call it "delaying spill" principle).

Design of the Guide-line Method. Combining the above three principles, this paper designs a guide-line method for power generation operation of multi-reservoir of inter basins. The general idea of the guide-line method is as follows: first, draw the guide-line of reservoir according the above three 
principles; then coordinate the power decisions of each reservoir by using the guide-line of each reservoir.

1) Draw the guide line of reservoir

Guide line of reservoir is a helper curve that is used to determine the optimal power decision of reservoir. Of cause it should simultaneously satisfy the above three principles.

From the above analysis, it can be seen that reservoir should keep running at a high water level to reduce energy loss of inflow. In theory, energy loss of inflow is the minimum when power output of reservoir in each time period takes the minimum decision output, the smaller of the minimum power output and the corresponding power output of the minimum release of reservoir, on condition that the storage capacity of reservoir is large enough. But in fact, the storage capacity of reservoir is limited and it will produce lots of spillage in the latter time periods when reservoir runs at the minimum decision output in each time period. Obviously it doesn't conducive to the full use of hydropower resource. So it is necessary to coordinate the "high water level" principle and the "reducing spillage" principle to find the highest water level line in non-spill situation. The specific method is as follows.

It can obtain a storage trajectory to make release of reservoir at each time period be equal to the maximum power flow of hydropower plant and recursive calculate from the last time period to the first one starting from the final target storage of reservoir according to the water balance equation. The storage trajectory is called the spill-preventing line of reservoir.

If annual run-off of reservoir is very sufficient or annual run-off converges on one or two time periods, spill cannot be avoid because there will be some water left after the reservoir is filled up. In this case, the spill-preventing line should be adjusted by moving the spillage to the latter time periods on condition that the total spillage keeps constant as the "delaying spill" principle described. In this way, water level of reservoir can be raised and energy loss of inflow can be reduced. The adjusted spill-preventing line is the highest storage trajectory in non-spill situation and can be used as the guide line for power generation.

2) Determine the power decision of reservoir by using guide-line

It can determine the power output of reservoir in the current time period after the guide line of reservoir is obtained. The determining process of time period $t_{0}$ by using the guide-line method is elaborated as follows.

As guide line is the highest storage trajectory of reservoir in non-spill situation, reservoir should always generate at the minimum decision power to quickly raise its water level to reduce energy loss of inflow as long as its storage of time period $t_{0}$ is lower than the guide line. However, if the ending storage of time period $t_{0}$ is higher than the guide line when reservoir generates at the minimum decision power, a coordination should be conducted between the "high water level" and "reducing spillage" principles to minimizing the total energy loss of reservoir system. Fig. 1 is a schematic for coordinating the "high water level" and "reducing spillage" principles in which, $V_{t_{0}}$ is the starting storage of time period $t_{0}$, in $\mathrm{m}^{3} ; E_{\text {loss }, t_{0}}$ and $\Delta E_{\text {spill, } t_{0}}$ denote energy loss of inflow and decrement of spill energy caused by increasing a unit power, in $\mathrm{kW} \cdot \mathrm{h} ; V_{t_{0}+1}^{*}$ is the ending storage of time period $t_{0}$ satisfying $E_{\text {loss }, t_{0}}=\Delta E_{\text {spill, } t_{0}}$, in $\mathrm{m}^{3}$.

In Fig. 1, as the storage of reservoir is higher than the guide line, spill cannot be avoided. In this case, reservoir should move the spillage to the latter time period as far as possible to raise the early water level according to the "delaying spill" principle. In Fig. 1, as storage of reservoir is always lower than the guide line during the process of delaying spill, the spillage is finally moved to time period $T$, the last time period. But in some situations, storage of reservoir in a middle time period $t_{\mathrm{s}}\left(t_{0}<t_{\mathrm{s}}<T\right)$ may have reached the storage upper limit during the process of delaying spill. In this case, the spillage should be moved to time period $t_{\mathrm{s}}$ and reservoir should run by the guide line at the remaining time periods. So the expressions of $E_{\text {loss, } t_{0}}$ and $\Delta E_{\text {spill, } t_{0}}$ are 


$$
\begin{aligned}
& E_{\text {loss }, t_{0}}=\frac{\Delta Z_{t_{0}}}{2} \cdot I_{t_{0}} \cdot \Delta t+\Delta Z_{t_{0}} \cdot \sum_{t=t_{0}+1}^{t_{\mathrm{e}}} I_{t} \cdot \Delta t \\
& \Delta E_{\text {spill } t_{0}}=\left(Z_{\mathrm{u}, t_{\mathrm{e}}}-\underline{Z}_{\mathrm{d}}\right) \cdot \Delta Y_{t_{\mathrm{e}}} \cdot \Delta t
\end{aligned}
$$

Where $\Delta Z_{t_{0}}$ denotes decrement of water level caused by increasing a unit power, in meter; $t_{\mathrm{e}}$ is the last time period for calculating energy loss of inflow and $t_{\mathrm{e}}=T$ or $t ; \Delta Y_{t_{\mathrm{e}}}$ is increment of spillage in time period $t_{\mathrm{e}}$ caused by increasing a unit power in time period $t_{0}$, in $\mathrm{m}^{3} / \mathrm{s}$.

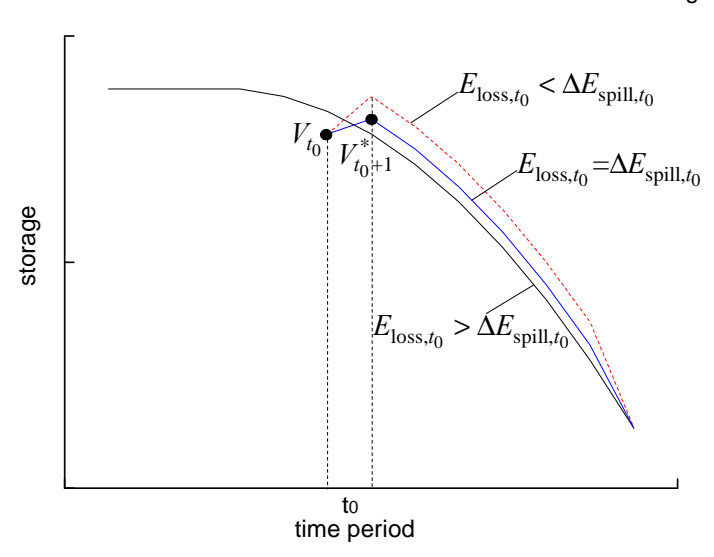

Fig. 1. Schematic of coordinating the "high water-level running" and "minimum spillage" principles.

In Fig. 1, as the ending storage of time period $t_{0}$ is higher than the guide line when reservoir runs at the minimum decision power in time period $t_{0}$, spill cannot be avoided and the spillage is finally moved to the time period $T$ according to the "delaying spill" principle. That is to say, reservoir runs at the maximum power flow during time period $t_{0}$ to time period $T-1$ and reduces its water level to the target water level at the end of time period $T$. If $E_{\text {loss }, t_{0}}<\Delta E_{\text {spill, } t_{0}}$, namely the decrement of spill energy is sufficient to make up for the energy loss of inflow, reservoir should increase it power output until $E_{\text {loss }, t_{0}}=\Delta E_{\text {spill, } t_{0}}$.

3) Coordinate power decision of each reservoir

In power generation operation of inter-basin multi-reservoir, the minimum power constraint of multi-reservoir is a bond that connects each reservoir and should be satisfied. If the minimum power constraint of multi-reservoir is broken when each reservoir runs by its own guide line, a coordination should be conducted among reservoirs. Assume that the power vacancy is $\Delta N$, then the purpose of coordination is to distribute $\Delta N$ among different reservoirs to maximizing the sum of energy loss of inflow and spill energy. The key of power distribution is calculating energy loss of inflow and spill energy.

$\Phi$ Time span for calculating energy loss

It will affect the storage state of reservoir in latter time periods to change the power output of reservoir in current time period. The affected time span is related to the ending water level of the current time period and the guide line of reservoir. To the guide line, the storage state of reservoir has two situations as Fig. 2 shown.

(a) of Fig. 2 shows the situation that the ending storage state of time period $t_{0}$ is lower than the guide line. In this situation, reservoir will first run at a constant water level from time $\mathrm{b}$ to time $\mathrm{c}$ and then run by the guide line from time $\mathrm{c}$ to the end of planning horizon to avoid spill. As power changing of time period $t_{0}$ will not affect the energy loss of inflow and spill energy of time span from time span $\mathrm{c}$ to the end of planning horizon, the time span for calculating energy loss is from time a to time $\mathrm{c}$.

(b) of Fig. 2 shows the situation that the ending storage state of time period $t_{0}$ is higher than the guide line. In this situation, spill cannot be avoided. According to the "delaying spill" principle, the 
spillage should be moved to the latter time periods as far as possible. Assume that the spillage is moved to time period $t_{\mathrm{e}}$ ( $t_{\mathrm{e}}=T$ or $t_{\mathrm{s}}$ according to the previous analysis). As reservoir will run by the guide line, energy loss of inflow and spill energy after time period $t_{\mathrm{e}}$ will not be affected by power changing of time period $t_{0}$. Hence, the time span for calculating energy loss is from time a to time $\mathrm{d}$ (the end of time period $t_{\mathrm{e}}$ ).

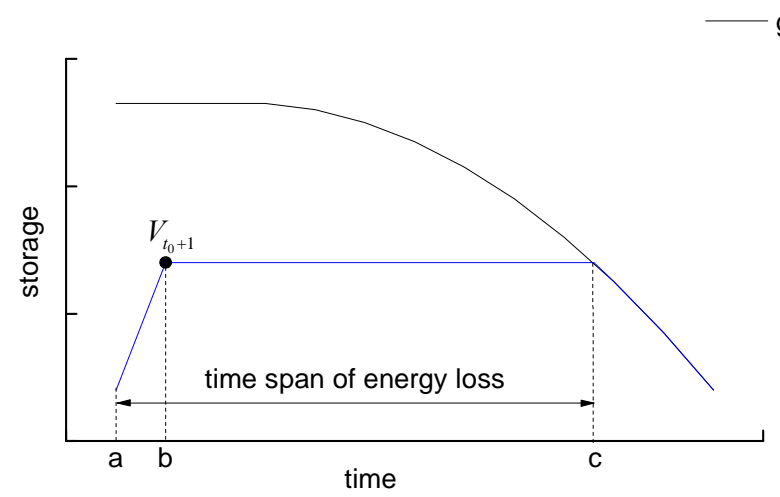

(a) The ending storage state of time period $t_{0}$ is lower than the guide line

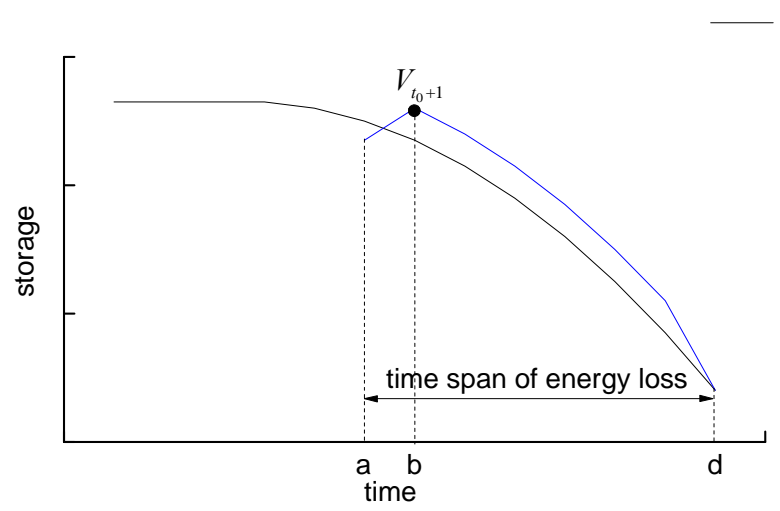

(b) The ending storage state of time period $t_{0}$ is higher than the guide line

Fig. 2. Schematic of determining the time range of energy loss of inflow.

Q Calculate energy loss of inflow

It can calculate the energy loss of inflow once the affected time span is determined. The calculating formula of energy loss of inflow caused by increasing a unit power in time period $t_{0}$ is

$$
E_{\text {loss }, t_{0}}=\frac{\Delta Z_{t_{0}}}{2} \cdot I_{t_{0}} \cdot \Delta t+\Delta Z_{t_{0}} \cdot \int_{\mathrm{b}}^{\text {end }} I(t) \cdot \mathrm{d} t
$$

Where $I(t)$ denotes inflow of reservoir in time period $t$, in $\mathrm{m}^{3} / \mathrm{s}$; "end" is the end time for calculating energy loss of reservoir. In Fig. 2, "end" is time c or d.

3 Calculate the decrement of spill energy

It will reduce the water level of reservoir and is conducive to reduce spillage to increase power output in the current time period. To the situation shown in (a) of Fig. 2, it is easily to understand that the decrement of spillage is equal to 0 when increasing power output in time period $t_{0}$; however, to the situation shown in (b) of Fig. 2, the spill energy of time period $t_{\mathrm{e}}$ will decrease when increasing power output in time period $t_{0}$. The decrement of spill energy can be calculated by Eq. (3).

Arithmetic Steps. The guide-line method for power generation operation of inter-basin multi-reservoirs consists of the following steps.

1) Determine the guide-lines of each reservoir.

2) Make the power generation scheme of the multi-reservoir system.

The method of determining the guide-line of reservoir introduced in step 1) includes the following substeps. 
Step1: determine the spill-preventing lines of each reservoir. The specific method is: make the releases of each reservoir in each time period equal to the maximum power outflow of reservoir and recursive calculate from the last time period to the first one starting from the final target water level. When the recursive calculation is over, a storage state sequence is obtained and it is the spill-preventing lines of reservoir. It should be noted that if the storage constraints of reservoir are broken in the above recursive calculation, the storage of the reservoir should take the boundary value.

Step2: determine the guide-line of each reservoir and the specific method is: check whether $Y_{t}>0$ for each $t(t=1,2,3 \ldots, T)$ when the reservoir running according to the spill-preventing line obtained in Step1. If no, the spill-preventing line is the guide-line to determine; otherwise, decrease $R_{t}$, the release of reservoir in time period $t$, and make $R_{t}$ equal to the maximum power outflow and move the spillage to the back time period as far as possible. When all the time periods are checked, the guide-line of reservoir is obtained.

The method of determining the power output of reservoir in time period $t$ introduced in step 2) includes the following substeps.

Step1: make $N_{t}$, the determination output of reservoir in time period $t$, equal to the minimum determination output of reservoir, then calculate $V_{t+1}$ according to the values of $N_{t}, V_{t}$ and $I_{t}$.

Step2: if $V_{t+1}$ is larger than the specified storage of guide-line, determine $N_{t}^{*}$, the optimal power output of reservoir in time period $t$, on the condition that $E_{\text {loss }, t}=\Delta E_{\text {spill, } t}$ by the method shown in Fig. 1 and make $N_{t}=N_{t}^{*}$; otherwise make $N_{t}$ equal to the minimum determination output of reservoir.

Step3: calculate $N_{\text {total }, t}$, the total power output of multi-reservoir system in time period $t$.

Step4: if $N_{\text {total, } t} \geq \underline{N}_{\text {total }, t}$, stop; otherwise, adjust the power outputs of each reservoir by the method introduced above to make the minimum power constraint of multi-reservoir system be satisfied.

Repeating Step1 to Step4 for each $t(t=1,2,3 \ldots, T)$, then the power outputs of each reservoir in each time period are determined and the power generation scheme of the multi-reservoir system is obtained.

\section{Case Study}

The inter-basin multi-reservoir system consisting of Dongjiang and Shuangpai reservoirs located on Xiang River and Jiangya and Zaoshi reservoirs located on Li River is taken as example to test the performance of the guide-line method. Xiang River is one of the seven branches of Yangtse River and is the latgest river in Hunan province. Dongjiang and Shuangpai are the key hydropower stations of Xiang River basin with total installed capacity of 710MW. Li River is the northernmost river among the four rivers of Hunan province, namely Xiang River, Zi River, Yuan River and Li River. Jiangya and Zaoshi are the key hydropower stations of Li River basin with total installed capacity of 420MW. These two rivers are used as an illustrative example because there are obvious differences between the hydrology characteristic of them.

The guide-line method, DPSA and the criminate criterion method are used to solve the multi-reservoir optimization problem, respectively. DPSA is a variant of DP and its solving process is: first, divide the N-dimensional optimization problem of multi-reservoir system into $\mathrm{N}$ onedimensional subproblems that each has less decision or state variables than the original problem; then, use DP to solve each subproblem; last iterates until convergence. DPSA effectively alleviates the "dimension curse" of DP and its computation and memory requirements grow linearly with N, the number of reservoirs. More details about DPSA are given by Opan [0] and Cheng et al. [0].

The characteristic parameters of each reservoir are given in Table 1 and the power generations and executing times of each method are given in Table 2. The water level processes of Dongjiang and Jiangya produced by the guide-line method and the criminate criterion method are shown in Fig. 3 and Fig. 4. 
Table 1. Characteristic parameters of reservoirs.

\begin{tabular}{ccccccc}
\hline Reservoir & $\begin{array}{c}\text { Adjusting } \\
\text { capability }\end{array}$ & $\begin{array}{c}\text { Normal water } \\
\text { level }[\mathbf{m}]\end{array}$ & $\begin{array}{c}\text { Dead water } \\
\text { level [m] }\end{array}$ & $\begin{array}{c}\text { Regulation } \\
\left.\text { storage [10 } \mathbf{~ m}^{\mathbf{3}}\right]\end{array}$ & $\begin{array}{c}\text { Installed } \\
\text { capacity [MW] }\end{array}$ & $\begin{array}{c}\text { Firm power } \\
\text { [MW] }\end{array}$ \\
\hline Dongjiang & Multi-yearly & 285 & 242 & 52.69 & 560 & 80.0 \\
Shuangpai & Seasonal & 170 & 158 & 3.74 & 150 & 1.75 \\
Jiangya & Annual & 236 & 188 & 11.60 & 300 & 37.1 \\
Zaoshi & Annual & 140 & 112 & 9.30 & 120 & 18.4 \\
\hline
\end{tabular}

Table 2. Result comparison of different methods

\begin{tabular}{ccccc}
\hline \multirow{2}{*}{ Item } & Reservoir & \multicolumn{3}{c}{ Method } \\
\cline { 3 - 5 } & & Criminate criterion & DPSA & Guide-line \\
\hline \multirow{2}{*}{ Power generation $[\mathrm{MW} \cdot \mathrm{h}]$} & Dongjiang & 772054 & 774012 & 772862 \\
& Shuangpai & 711890 & 714838 & 710378 \\
& Jiangya & 621174 & 652440 & 656200 \\
& Zaoshi & 278992 & 295505 & 302355 \\
\hline Spillage $\left[10^{6} \mathrm{~m}^{3}\right]$ & Sum & 2389690 & 2436795 & 2441795 \\
Time $[\mathrm{s}]$ & Sum & 4121 & 3666 & 3554 \\
\end{tabular}

Note: The number of disperse states is 600 ; the clock speed of CPU is $2.13 \mathrm{GHz}$ and the memory capacity is $2 \mathrm{~GB}$.

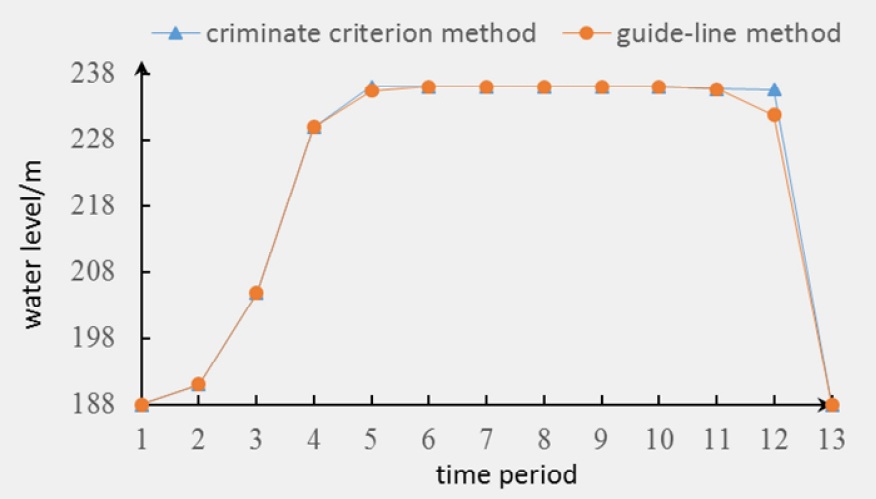

Fig. 3. Monthly water level of Jiangya reservoir.

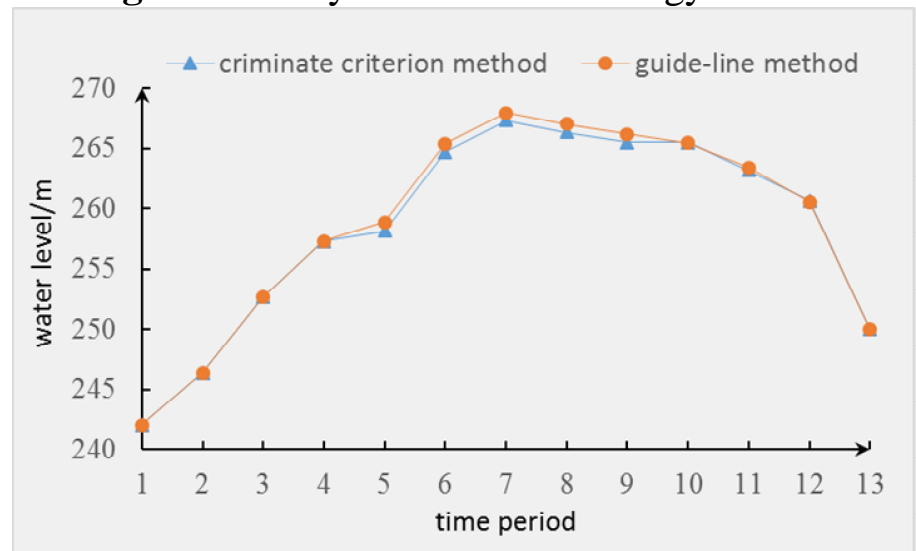

Fig. 4. Monthly water level of Dongjiang reservoir.

From Table 2, it can been seen that the executing time of the guide-line method is slightly larger than the discriminant criterion method, but the spillage of the former is 0.567 billion $\mathrm{m}^{3}$ less than that of the latter and the power generation of the former is $52105 \mathrm{MW} \cdot \mathrm{h}(2.18 \%)$ larger than the latter. These results show that the criminate criterion method has small calculation but is easy to produce spillage when applying to multi-reservoir operation; however, the guide-line method improves the solving efficiency of the multi-reservoir optimization problem and makes up for the defects of the criminate criterion method.

From Table 2, it can be seen that the executing time of the guide-line method is $99 \%$ shorter than DPSA. The reason is that much knowledge and experiences of generation operation of reservoir are blended into the guide-line method. In order to obtain the optimal operation scheme of the 
multi-reservoir system, the only thing needs to do is that draw the guide lines of each reservoir and coordinate the power generating decisions of each reservoir by using the guide line. The drawing and applying of the guide line of reservoir is simple and easily to achieve. It avoids the complex enumeration and tedious calculating and comparing of power generations. Besides, the power generation of the multi-reservoir system produced by the guide-line method is $0.21 \%$ larger than that produced by DPSA (see Table 2). These results demonstrate that the guide-line method can obtain relatively satisfactory solution to the optimization problem of multi-reservoir operation.

Fig. 3 shows the water level trajectories of Jiangya reservoir produced by the discriminant criterion method and the guide-line method. From Fig. 4, it can be seen that Jiangya reservoir kept its water level constant until the last time period after reaching the highest water level and emptied itself during the last time period according to the discriminant criterion method, which produced a lot of spillage; however, the guide-line method avoided the spill problem by emptying the reservoir during the last two time periods. The water level trajectories of Zaoshi reservoir produced by these two methods have similar law to Fig. 3.

Fig. 4 shows the water level trajectories of Dongjiang reservoir produced by the discriminant criterion method and the guide-line method. From Fig. 4, it can be seen that the water level trajectory produced by the discriminant criterion method is lower than the guide-line method. The reason is that the K-value of Dongjiang reservoir according to the discriminant criterion method is smaller than that of any other reservoirs through the whole planning horizon. According to the storing and supplying principles of the discriminant criterion method, Dongjiang reservoir always stores water behind other reservoir and supplies water before other reservoir. As a result, the water level of Dongjiang reservoir becomes lower and lower over time. It may cause premature emptying of the reservoir when encountering dry season, which is bad for safety and economy running of reservoir.

These results demonstrate that although taking account of the influence of raising water level on power generation, the discriminant criterion method may lead to large amount of water abandoned as it ignores reservoir spill. However, the guide-line method avoid the centrally storing or supplying problem of the discriminant criterion method and decreases reservoir spillage by taking full account of energy loss of inflow as well as abandoned energy caused by storing or supplying of reservoir.

In conclusion, the guide-line method for generation operation of inter-basin reservoirs presented in this paper is feasible and effective.

\section{Conclusion}

This paper summarizes the knowledge and experiences of generation operation of reservoir from the view of energy conversion and proposed a guide-line method for generation operation of inter-basin reservoirs by combines the knowledge and experiences with optimization theory. The simulating results show that the guide-line method has high accuracy and efficiency and has advantages on both execution time and optimization degree over DPSA. An outstanding feature of the guide-line method is that it overcomes the defect of emptying reservoir and abandoning water of the discriminant criterion method.

The guide-line method is an optimization method that blends the knowledge and experiences of generation operation of reservoir into optimization algorithm. It offers a novel ideal for solving the optimization problem of multi-reservoir operation and has theoretical and practical application value.

\section{Acknowledgments}

This work is supported by National Natural Science Foundation of China (51379081) and State Grid Corporation of China (DKJS-13-00220).

\section{References}

[1] J. F. Wu: Sci-Tech \& Development of Enterprise Vol. (2008), p. 169-171. 
[2] A. Y. Chen, Y. B. Chen, W. X. Zhang: Journal of China Three Gorges University Vol. 17 (1995), p. $77-85$.

[3] T. T. G. Zhao, X. H. Lei, Y. Z. Jiang and H. Wang: Journal of Hydraulic Engineering Vol. 43 (2012), p. 414-421.

[4] C. M. JI, C. G. Li, X. Y. Liu, B. Q. Wang and P. Zhang: Journal of Hydraulic Engineering Vol. 47 (2016), p. 1-9.

[5] Z. B. Zhang, X. C. Wu, S. H. Zhang, Y. Z. Jiang, S. M. Geng and H. Wang: Journal of Hydroelectric Engineering Vol. 33 (2014), p. 21-27.

[6] Y. Q. Zhao: Journal of Hohai University Vol. 19 (1991), p. 53-58.

[7] A. Q. Li, L. P. Wang, C. M. Ji and B. Xiang: Hydropower Automation and Dam Monitoring Vol. 31 (2007), p. 5-9.

[8] S. Ou, J. Z. Zhou, C. Zhou and H. Wang: Journal of Hydraulic Engineering 44 (2013), 435-443.

[9] Y. P. Miao, P. Wei, F. X. Chen and Y. S. Zhan: Water Resources and Power Vol. 32 (2014), p. 49-52.

[10] Y. Y. Li, J. Z. Zhou, S. Q. Zhang, C. L. Huang and D. Yan: Journal of Hydroelectric Engineering Vol. 35 (2016), p. 32-40.

[11] M. Heidari, V. T. Chow, P. V. Kokotovic and D. D. Meredith: Water Resources Research Vol. 7 (1971), p. 273-282.

[12]Z. K. Feng, S. L. Liao, W. J. Niu, C. T. Cheng, J. X. Tang, H. Y. Su: Proceedings of the CSEE Vol. 35 (2015), p. 4635-4644.

[13]M. Opan: Teknik Dergi Vol. 22 (2011), p. 5359-5385.

[14]C. T. Cheng, F. Y. Yang, X. Y. Wu and H. Y. Su: Journal of Hydroelectric Engineering Vol. 29 (2010), p. 71-77.

[15]H. R. Howson and N. G. F. Sancho: Math. Programming Vol. 8 (1975), p.104-116.

[16]C. Zhang, J. Z. Zhou, C. Wang, Y. C. Zhang and L. Mo: Journal of Hydroelectric Engineering Vol. 35 (2016), p. 12-21.

[17]C. M. Ji, Z. Q. Jiang, P. Sun, B. K. Zhao and T. Zhou: Journal of Hydroelectric Engineering Vol. 33 (2014), p. 118-125.

[18] M. Zhang, Y. Ding, X. H. Yuan and C. J. Li: J. Huazhong Univ. of Sci. \& Tech. Vol. 34 (2006), p. $90-92$.

[19]Z. Y. Pei, Y. G. Wu, C. M. Ji and G. C. Li: Automation of Electric Power Systems Vol. 34 (2010), p. 23-26.

[20]X. Y. Zhou, Y. W. Ma, Y. C. Zhang: Journal of Hydraulic Engineering Vol. 30 (1999), p. 1-9. 